\title{
Hypoxia and temporal aspects of hypothalamic self-stimulation
}

\author{
MARTIN J. GERBEN, $U . S$. ARMY RESEARCH INSTITUTE OF \\ ENVIRONMENTAL MEDICINE, Natick, Mass. 01760
}

Six rats were trained to operantly control the duration of electrical stimulation to the posterior hypothalamus. The duration of the stimulus was equal to the duration of the lever deflection response. Under normoxic conditions $\left(21 \% 0_{2}\right)$, increasing stimulus current levels yielded increasing response rates, decreasing response durations and decreasing inter-response times. Hypoxia $\left(8 \% \mathrm{O}_{2}\right)$ did not affect response duration at any current level, but lengthened inter-response time. The magnitude of this effect was inversely related to current level. Results are interpreted as suggesting that hypoxia does not affect the neural response concurrent with electrical stimulation but affects the after-effects of stimulation.

Annau \& Weinstein (1967) have recently demonstrated an interaction between atmospheric oxygen concentration and stimulus current level on the rate of operant responding reinforced by brief electrical stimulation of the posterior hypothalamus. At low stimulus current levels, even mild hypoxia greatly reduced response rate. At high current levels, no decrement in response rate was associated with mild hypoxia and relatively little decrement was associated with more severe levels of hypoxia.

These authors proposed a neural model of the effects of hypoxia in order to explain their results. Noting that both hypoxia and the lowering of stimulus current level have similar decremental effects on response rate, Annau and Weinstein hypothesized similar neural effects of the variables. Specifically, both hypoxia and the lowering of stimulus current levels were hypothesized to reduce the number of neurons reacting to the electrical stimulus.

This model does not specify whether hypoxia is having its effect on neural events occurring in the presence of electrical stimulation or on neural events occurring during the interval between the presentation of the electrical stimuli. Such an inference was not possible on the basis of Annau and Weinstein's data because their one behavioral measure, response rate, provides no information concerning the differential effects of hypoxia during and after electrical stimulation.

An experimental procedure which yields such information involves S's operant regulation of the duration of the stimulus pulse train (Stein, 1962; Keesey, 1964). Rats that will deflect a lever to initiate hypothalamic stimulation will also release the lever to terminate the stimulation. The time between the deflection response, which yields the onset of stimulation, and the release response, which yields the offset of stimulation, may be assumed to be a reflection of the neural response during stimulation. Similarly, the time between the release response, which yields the offset of one stimulus, and the deflection response, which yields the onset of the next stimulus, may be assumed to be a reflection of neural processes occurring between stimuli. The present experiment measures both these aspects of behavior in order to assess the contribution of each to the interaction between hypoxia and stimulus current level on response rate.

Subjects. Six male Sprague-Dawley rats with bipolar electrodes implanted to the posterior hypothalamus served as Ss. The stereotaxic coordinates for implantation were $3.25 \mathrm{~mm}$ posterior to the bregma, $0.75 \mathrm{~mm}$ lateral to the midline, and $9.00 \mathrm{~mm}$ below the surface of the skull. Ss were allowed two weeks recovery after surgery and were maintained on ad lib schedules of food and water during the experiment.

Apparatus. A plastic vacuum dessicator, modified to allow gas mixing and fitted with a rat lever, served as an operant conditioning chamber. Cylinderized $0_{2}-\mathrm{N}_{2}$ mixtures provided the experimental atmospheres. At a flow rate of $20 \mathrm{l} / \mathrm{min}$ the chamber could be flushed within $3 \mathrm{~min}$. A sound proof outer chamber provided isolation from extraneous stimuli. A stimulator provided biphasic square wave pulse pairs $(0.1 \mathrm{msec}$ duration, $0.1 \mathrm{msec}$ inter-pulse interval, $100 \mathrm{pps}$ ). Temporal measurements were made using solid state programming equipment and electro-mechanical clocks which read to the nearest $0.1 \mathrm{sec}$.

Design and Procedure. A treatments $\left(21 \% 0_{2}\right.$ and $8 \% 0_{2}$ atmospheres) by treatments (zero to peak current levels of 1.6,2.0, 2.4, and $2.8 \mathrm{~mA}$ ) by Ss design was used. Ss were pretrained in the operant conditioning chamber in an atmosphere of room air. Five 1 th pretraining sessions were presented on five consecutive days. Each lever deflection was reinforced by an electrical stimulus to the hypothalamus. The duration of this stimulus was equal to the duration of the lever deflection. During each pretraining session, responding was allowed for $15 \mathrm{~min}$ under each of the four current levels, the order of presentation being randomly determined.

Experimentation occurred on the sixth day of training. The experimental session consisted of four phases. Phase 1 was $5 \mathrm{~min}$ in duration and allowed the introduction of the $21 \% 0_{2}$ atmosphere at a flow rate of $201 / \mathrm{min}$. During Phase 1, operant responding was initiated, the current level being equal to the initial current level subsequently being presented during Phase 2 . After $5 \mathrm{~min}$ had elapsed, Phase 2 was begun. During this phase, 125 responses were allowed under each of the four current levels, the order of presentation of the current levels being randomized. Phase 3 was identical to Phase 1, except that the $8 \% \mathrm{O}_{2}$ atmosphere was introduced. Phase 4 was identical to Phase 2, except that the $8 \% 0_{2}$ condition was maintained.

Three temporal aspects of behavior were measured: (1) the total time (TT) required to complete the last 100 responses under each experimental

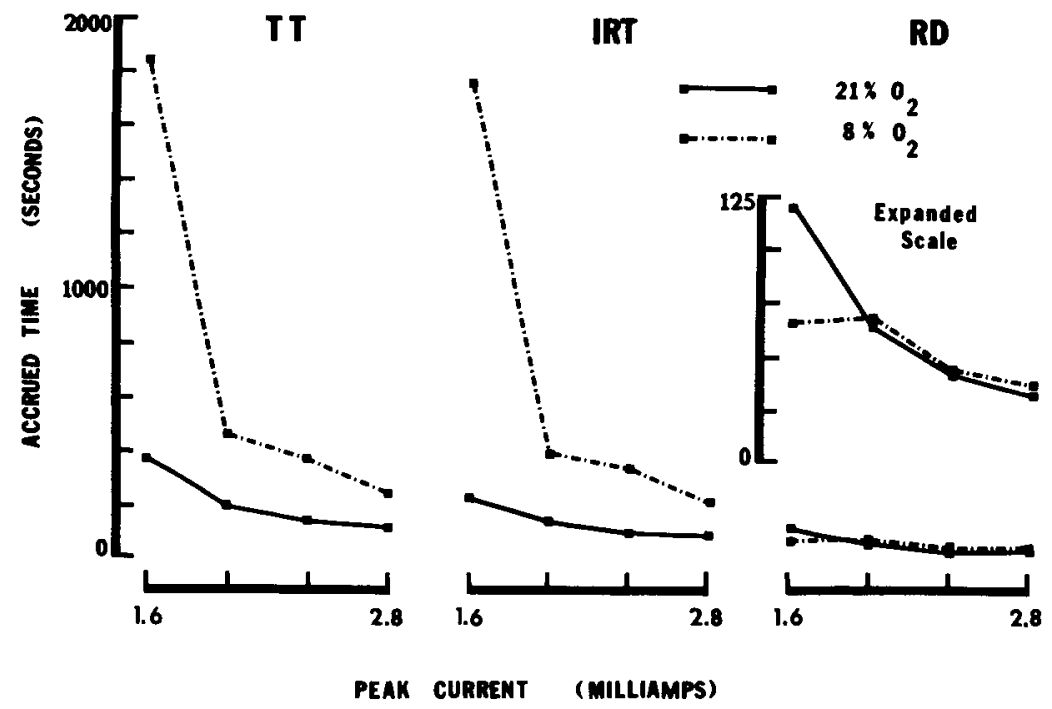

Fig. 1. Mean time in seconds accrued during TT, IRT, and RD as a function of oxygen and current levels. Note the similarity between TT and IRT. The apparent difference between the $8 \%$ and $21 \% 0_{2}$ values of RD under conditions of $1.6 \mathrm{~mA}$ was not statistically significant. 
condition; (2) the time accrued during the corresponding 100 inter-response times (IRT); (3) the time accrued during the corresponding 100 response durations (RD). The first 25 responses under each condition were eliminated from the data in order to minimize contamination of the data by transient carry-over effects resulting from the previous stimulus current level.

Results. Mean TT, IRT, and RD under each experimental condition are plotted in Fig. 1. All measures evidenced considerable heterogeneity of variance. Therefore, a logarithmic transformation of the data was used in an analysis of variance. Inspection of Fig. 1 and the analysis of variance indicate as current level increased, TT decreased $(\mathrm{F}=17.14, \mathrm{df}=3 / 15, \mathrm{p}<.001)$, IRT decreased $(F=15.20, \mathrm{df}=3 / 15, \mathrm{p}<.001)$, and $\mathrm{RD}$ decreased $(\mathrm{F}=$ $10.69, \mathrm{df}=3 / 15, \mathrm{p}<.001)$. Hypoxia increased TT $(F=30.29$, df $=1 / 5 \mathrm{p}<.005)$ and IRT $(\mathrm{F}=38.80, \mathrm{df}=1 / 5, \mathrm{p}<.005)$, but had little effect on RD. In addition, oxygen and current levels interacted with respect to both TT $(\mathrm{F}=3.90, \mathrm{df}=3 / 15, \mathrm{p}<.01)$ and IRT $(F=4.94, \mathrm{df}=3 / 15, \mathrm{p}<.025)$. Hypoxia had a much greater effect at the lower current levels. No significant interaction occurred with respect to RD. In the expanded scale panel of Fig. 1 , the apparent difference between the $8 \%$ and $21 \% 0_{2}$ RD values under the $1.6 \mathrm{~mA}$ stimulus conditions was not statistically significant.

Discussion. Since TT is reciprocally related to response rate, the present results replicate those of Annau and Weinstein. Hypoxia and the reduction of stimulus current level both reduced response rate. The decremental effect of hypoxia was potentiated as current level decreased. This replication emphasizes the generality of these effects since they occurred in spite of certain differences between the present experiment and those of Annau and Weinstein. Firstly, Annau and Weinstein used 60 cycle sine wave current while biphasic square wave pulses were used in the present experiment. Secondly, Annau and Weinstein used a fixed stimulus duration of $0.25 \mathrm{sec}$ while the present procedure allowed $S$ to control the duration of stimulation. Thirdly, Annau and Weinstein used Wistar rats while the present Ss were from the Sprague-Dawley strain.

Changes in response rate associated with hypoxia were correlated with changes in IRT rather than changes in RD. That is, the increases in TT (i.e., the decrease in response rate) at each current level under hypoxic conditions were entirely attributable to similar increases in IRT. In addition, hypoxia acted similar to a reduction in current only during IRT, when stimulation was not being presented. While the reduction of stimulus current level increased $\mathrm{RD}$, hypoxia had little effect on this aspect of self-stimulation behavior. Thus, hypoxia did not act similar to a reduction in current during RD when the electrical stimulus was being presented. The increases in TT associated with decreasing current levels can be contrasted to those increases in TT resulting from exposure to hypoxia, the former being correlated with increases in both IRT and RD, the latter being correlated only with increases in IRT.

The present results are contrary to what would be expected if the reduction in response rate associated with hypoxia was mediated by the same neural mechanism as was the reduction in response rate associated with the lowering of stimulus current levels. The difference between the effects of oxygen and current level on RD suggests that hypoxia did not affect neural reactions concurrent with electrical stimulation while current level affected these reactions. Conceivably, hypoxia may not have affected neural reactions at or near the site of stimulation.

The similarity of the effects of hypoxia on IRT to those resulting from the reduction of stimulus current level suggests that both oxygen and current levels affected neural events occurring after the termination of stimulation. In this regard, neural after-effects of stimulation of the posterior hypothalamus occur in several areas of the brain, including the cortex (Gellhorn, 1957), an area whose functional integrity is especially sensitive to hypoxia (Sugar \& Gerard, 1938). Thus, the interaction between oxygen and current levels on the rate of hypothalamic self-stimulation may be related to an interaction of these variables occurring after the offset of stimulation in brain areas other than the site of stimulation.

\section{REFERENCES}

ANNAU, Z., \& WEINSTEIN, S. A. Hypothalamic self-stimulation: interaction of hypoxia and stimulus intensity. Life Sciences, 1967, 6, 1355-1360.

GELLHORN, E. Autonomic imbalance and the hypothalamus. Minneapolis, Minn.: Univ. Minnesota Press, 168-216.

KEESEY, R. E. Duration of stimulation and the reward properties of hypothalamic stimulation. J. comp. physiol. Psychol., 1964, 58, 201-207.

STEIN, $\mathbf{L}$. An analysis of stimulus-duration preference in self-stimulation of the brain. J. comp. physiol. Psychol, 1962, 55, 405-414.

SUGAR, O., \& GERARD, R. W. Anoxia and brain potentials. J. Neurophysiol., 1938, 1, 558-572. 